\title{
基于凸优化的举高消防车时间最优轨迹规划 ${ }^{*}$
}

\author{
滕儒民 李玉金王王 欣 赵 哲 \\ (大连理工大学机械工程学院 大连 116024)
}

\begin{abstract}
摘要: 为提高举高消防车作业的及时性、可靠性与安全性, 提出一种考虑动作平稳、无冲击的时间最优轨迹规划算法。引入 伪位移参量 $s$ 表示路径, 利用 $\mathrm{B}$ 样条曲线对举高消防车臂架系统各个关节运动离散点进行拟合, 构造连续运动几何路径。结 合连续路径, 以 $s$ 及其对于时间的各阶导数表示时间最优目标函数与各个关节速度、加速度约束, 建立时间最优轨迹规划的 凸优化模型。利用 $\mathrm{B}$ 样条曲线以有限维矢量 $\boldsymbol{x}$ 表示轨迹, 采用内点法对凸优化模型进行求解, 在保证速度、加速度、加加速 度连续的条件下求得最优解, 进而得到各个关节的运动轨迹。利用提出的方法对大高度举高消防车进行轨迹规划, 经仿真表 明, 在满足动作平稳性的同时得到时间最优运动轨迹。
\end{abstract}

关键词: 举高消防车; 轨迹规划; 时间最优; 凸优化; B 样条曲线

中图分类号: $\mathrm{TH} 218$

\section{Time Optimal Trajectory Planning of Elevating Fire Truck Based on Convex Optimization}

\author{
TENG Rumin LI Yuxin WANG Xin ZHAO Zhe
}

(School of Mechanical Engineering, Dalian University of Technology, Dalian 116024)

\begin{abstract}
In order to improve the timeliness, reliability and security of elevating fire truck, a time optimal trajectory planning algorithm is proposed, considering the stability and non-impact of motions. Pseudo-displacement $s$ is introduced, to fit discrete points of joints motion and construct continuous geometric path with B-spline. A convex optimization model is established, which is based on the continuous path, time optimal objective function that is formulated with $s$ and its derivatives of time, as well as joint velocity and acceleration constraints. B-spline is utilized to parameterize trajectories with finite-dimensional vector $\boldsymbol{x}$, and interior point method is used to solvethe convex optimization model, to get optimal trajectories of joints, while guaranteeing that velocities, accelerations and jerks are continuous. The proposed algorithm is simulated on a high elevating fire truck, and the results demonstrate that time optimal trajectory is obtained while the stability of motion is satisfied.
\end{abstract}

Key words: elevating fire truck; trajectory planning; time optimal; convex optimization; B-spline

\section{0 前言}

举高消防车用于高层建筑的救援与消防作业。 由于工作环境的特殊性与严峻性, 要求臂架的动作 快速、平稳、无冲击, 以确保救援与消防作业的及 时性、可靠性与安全性。尤其对于大高度举高消防 车, 动作多样并且操作复杂, 为保证作业高效可靠, 进行时间最优轨迹规划的研究十分必要。

通过在关节空间对臂架系统的关节组合运动进 行以时间最优与动作平稳为目标的轨迹规划, 可得 到臂架系统的时间最优运动轨迹, 满足各个关节的

* 辽宁省高校创新团队支持计划资助项目(LT2014001)。20180322 收到初 稿, 20180905 收到修改稿
动作平稳性, 减小臂架动作引起的冲击与振动。

轨迹规划是在几何路径限制的基础上对多关节 机器人进行时间序列的生成, 进而计算各个关节的 运动轨迹 ${ }^{[1]}$ 。而时间最优轨迹规划则在此基础上以 动作时间最短为目标, 构造时间最短目标函数, 并 施加多个影响运动性能的约束条件, 以规划满足多 方面需求的运动轨迹。对其进行的研究已产生多样 的成果, 如以相平面 $s-\dot{s}$ 为基础的动态规划法 ${ }^{[2] 、}$ 线性规划法 ${ }^{[3]}$, 通过构造数学曲线进行的混合遗传/ 区间算法 ${ }^{[4]}$ 、分层式神经模糊推理 ${ }^{[5]}$ 、粒子群优化算 法 $^{[6]}$, 及结合 B 样条曲线的平坦法 ${ }^{[7]}$ 、改进的自适 应动态规划法 ${ }^{[8]}$ 等。此外, LIU 等[9-10]将时间最优 轨迹规划问题转化为凸优化问题, 并以优化解法求 得满足要求的最优轨迹。 
举高消防车臂架系统与普通多关节机器人在结 构方面有所区别, 虽然具有与多关节机器人旋转关 节类似的变幅关节, 但是还具有其不具备的伸缩关 节。而且不同于多关节机器人的伺服电机驱动控制 方式, 举高消防车各个关节以液压缸进行驱动, 以 电液伺服阀进行控制, 对此类臂架系统进行的轨迹 规划研究较少 ${ }^{[11-13] 。}$

本文以举高消防车为研究对象, 提出一种时 间最优轨迹规划算法, 考虑各个关节速度与加速 度约束, 将时间最优轨迹规划问题转化为凸优化 问题, 利用凸优化模型的特性求得全局时间最优 运动轨迹。经算例仿真, 该算法的有效性得到 证明。

\section{1 连续路径构造}

举高消防车结构如图 1 所示。根据实际工作情 况, 举高消防车臂架系统的运动可简化为 4 个自由 度, 即一号臂伸缩、二号臂变幅、二号臂伸缩、飞 臂变幅。一号臂的变幅角度固定为 $85^{\circ}$ 仰角。各个 自由度由液压缸驱动。对于伸缩关节, 伸缩关节线 位移为液压缸伸缩量; 对于变幅关节, 变幅关节角 位移与液压缸伸缩量呈非线性映射关系。

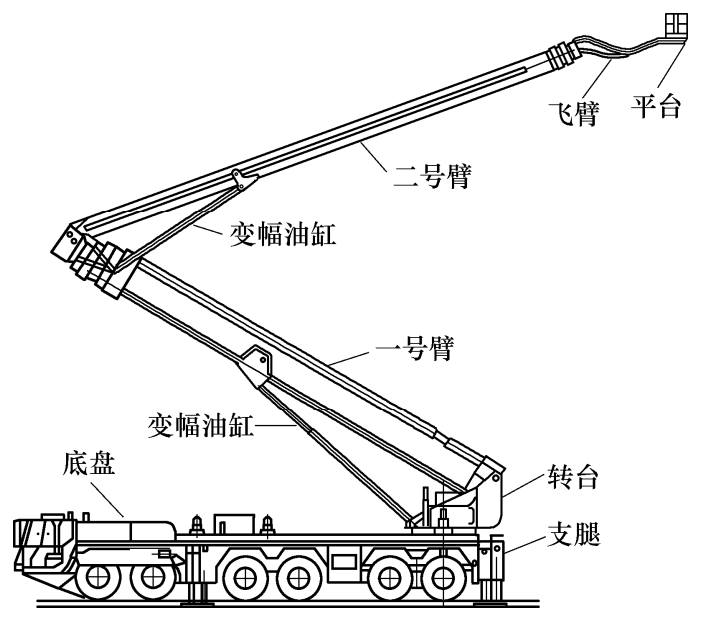

图 1 大高度举高消防车

考虑举高消防车的避障需求, 通过路径规划 ${ }^{[14]}$, 得到臂架系统各个关节运动需要经过的离散点序 列, 记为 $\boldsymbol{P}, \boldsymbol{P} \in \mathbf{R}^{n+k+1,4}$ 。

以伪位移参量 $s$ 表示末端平台路径, 关节空间 的 4 个关节变量以矢量 $\boldsymbol{q}(s)$ 表示, $\boldsymbol{q}(s) \in \mathbf{R}^{1,4}$ 。以 $s(t)$ 表示坐标轴 $s$ 与时间 $t$ 的函数关系, 通过 $\boldsymbol{q}(s(t))$ 实现 $\boldsymbol{q}(s)$ 与 $\boldsymbol{q}(t)$ 的相互转化。假定轨迹起始于 $t=0$, 结 束于 $t=T$, 约定 $s(0)=0$ 与 $s(T)=1$ 。根据伪位移物 理意义, 有 $0 \leqslant s(t) \leqslant 1$, 且对于任意 $t \in(0, T)$, 有 $\dot{s}(t)>0$ 。
对于离散点序列 $\boldsymbol{P}$, 采用 $k$ 阶 $\mathrm{B}$ 样条曲线构造 连续路径, 这样连续路径就继承了 $\mathrm{B}$ 样条曲线凸包 性、 $C^{k-1}$ 连续、局部支承等优良特性。由此得到的 路径平滑、多阶连续、易于修改, 并且方便表示与 控制，便于结合凸优化模型进行规划计算。

已知 $\boldsymbol{P} \in R^{n+k+1,4}$, B 样条曲线阶次为 $k$, 则控 制顶点个数为 $n+k+1$, 节点个数为 $n+2 k+2$ 。控 制顶点即为 $\boldsymbol{P}$, 设节点矢量为 $\boldsymbol{u}$, 取

$$
\begin{gathered}
\boldsymbol{u}=[\underbrace{s_{0}, \ldots, s_{k}}_{k+1}, s_{k+1}, \ldots, s_{p}, \ldots, s_{n+k}, \underbrace{s_{n+k+1}, \ldots, s_{n+2 k+1}}_{k+1}]= \\
{[\underbrace{0, \ldots, 0}_{k+1}, s_{k+1}, \ldots, s_{p}, \ldots, s_{n+k}, \underbrace{1, \ldots, 1}_{k+1}]}
\end{gathered}
$$

前 $k+1$ 与后 $k+1$ 节点取为重节点, 以确保离 散点序列经 $\mathrm{B}$ 样条曲线拟合后始末位置 ${ }^{[2]}$ 仍保持 不变。

$k$ 阶 B 样条曲线表示形式如下

$$
\boldsymbol{q}(s)=\sum_{j=i-k}^{i} P_{j} N_{j}^{k}(s) \quad i=k, \cdots, n+k
$$

式中, $\boldsymbol{P}_{j} \in \mathbf{R}^{1,4}$, 表示离散时刻 $t_{j}$ 对应的 4 个关节 值; $N_{j}^{k}(s)$ 为 $k$ 次规范 $\mathrm{B}$ 样条基函数。

$\boldsymbol{P}_{j}$ 已知。对于 $N_{j}^{k}(s)$, 有

$$
\begin{gathered}
N_{j}^{0}(s)=\left\{\begin{array}{ll}
1 & s_{j} \leqslant s<s_{j+1} \\
0 & \text { 其他 }
\end{array} \quad j=0,1, \cdots, n+2 k\right. \\
N_{j}^{r}(s)=\frac{s-s_{j}}{s_{j+r}-s_{j}} N_{j}^{r-1}(s)+\frac{s_{j+r+1}-s}{s_{j+r+1}-s_{j+1}} N_{j+1}^{r-1}(s) \\
j=0,1, \cdots, n+2 k-r, \quad r=1,2, \cdots, k
\end{gathered}
$$

利用 De Boor 公式计算 $\boldsymbol{q}(s)$ 对 $s$ 的 $r$ 阶导

$$
\begin{aligned}
& \boldsymbol{q}^{r}(s)=\sum_{j=i-k+r}^{i} \boldsymbol{P}_{j}^{r} N_{j}^{k-r}(s) \quad i=k, \cdots, n+k \\
& \boldsymbol{P}_{j}^{l}=\left\{\begin{array}{cc}
\boldsymbol{P}_{j} & l=0 \\
(k-l+1) \frac{\boldsymbol{P}_{j}^{l-1}-\boldsymbol{P}_{j-1}^{l-1}}{s_{j+k-l+1}-s_{j}} & j=i-(k-l), \cdots, i
\end{array}\right.
\end{aligned}
$$

则有

$$
\begin{aligned}
& \boldsymbol{q}^{\prime}(s)=\sum_{j=i-k+1}^{i} \boldsymbol{P}_{j}^{1} N_{j}^{k-1}(s) \\
& \boldsymbol{q}^{\prime \prime}(s)=\sum_{j=i-k+2}^{i} \boldsymbol{P}_{j}^{2} N_{j}^{k-2}(s) \\
& \boldsymbol{q}^{\prime \prime \prime}(s)=\sum_{j=i-k+3}^{i} \boldsymbol{P}_{j}^{3} N_{j}^{k-3}(s)
\end{aligned}
$$

式中, $\boldsymbol{q}^{\prime}(s)=\frac{\mathrm{d} \boldsymbol{q}(s)}{\mathrm{d} s}, \boldsymbol{q}^{\prime \prime}(s)=\frac{\mathrm{d} \boldsymbol{q}^{\prime}(s)}{\mathrm{d} s}, \boldsymbol{q}^{\prime \prime \prime}(s)=\frac{\mathrm{d} \boldsymbol{q}^{\prime \prime}(s)}{\mathrm{d} s}$ 。 


\section{2 凸优化模型建立}

在一组等式或不等式约束下, 求函数的最小值 (或最大值), 如果目标函数或约束条件中包含非线 性函数, 称这种规划问题为非线性优化问题。其一 般表示形式为

$$
\begin{array}{ll} 
& \min f(x) \\
\text { s.t } & C(x) \leqslant 0 \\
& \text { Ceq }(x)=0
\end{array}
$$

假定 $f(x) 、 C(x)$ 与 $C e q(x)$ 均为凸函数, 这样 的非线性优化称为凸优化。凸优化的可行域为凸集, 其局部最优解为全局最优解。

对于举高消防车的时间最优轨迹规划, 总动作 时间

$$
T=\int_{0}^{T} \mathrm{~d} t=\int_{s(0)}^{s(T)} \frac{\mathrm{d} t}{\mathrm{~d} s} \mathrm{~d} s=\int_{0}^{1} \frac{1}{\dot{s}} \mathrm{~d} s
$$

则时间最优轨迹规划的目标函数为

$$
T_{\min }=\min T=\min \int_{0}^{1} \frac{1}{\dot{S}} \mathrm{~d} s
$$

故具有 4 个关节变量的时间最优轨迹规划问题 转化为具有一个变量 $S$ 的时间最优轨迹规划问题。 由于目标函数为非线性函数, 此规划属于非线性优 化的范畴。

对于关节位移 $\boldsymbol{q}(s)$, 关节速度、加速度及加加 速度可通过链式法则推导得到

$$
\begin{gathered}
\dot{\boldsymbol{q}}(s)=\boldsymbol{q}^{\prime}(s) \dot{s} \\
\ddot{\boldsymbol{q}}(s)=\boldsymbol{q}^{\prime}(s) \ddot{s}+\boldsymbol{q}^{\prime \prime}(s) \dot{s}^{2} \\
\dddot{\boldsymbol{q}}(s)=\boldsymbol{q}^{\prime}(s) \dddot{s}+3 \boldsymbol{q}^{\prime \prime}(s) \dddot{s} \ddot{s}+\boldsymbol{q}^{\prime \prime \prime}(s) \dot{s}^{3}
\end{gathered}
$$

式中, $\dot{\boldsymbol{q}}(s)=\frac{\mathrm{d} \boldsymbol{q}(\mathrm{s})}{\mathrm{dt}}, \ddot{\boldsymbol{q}}(\mathrm{s})=\frac{\mathrm{d} \dot{\boldsymbol{q}}(\mathrm{s})}{\mathrm{dt}}, \dddot{\boldsymbol{q}}(\mathrm{s})=\frac{\mathrm{d} \ddot{\boldsymbol{q}}(\mathrm{s})}{\mathrm{dt}}$ 。 令

$$
\begin{gathered}
a(s)=\dot{s}^{2} \Rightarrow \dot{s}=\sqrt{a(s)} \\
b(s)=\ddot{s} \Rightarrow \ddot{s}=b(s) \\
d(s)=\frac{\dddot{s}}{\dot{s}} \Rightarrow \dddot{s}=d(s) \sqrt{a(s)}
\end{gathered}
$$

有关系式

$$
\begin{gathered}
b(s)=\frac{1}{2} a^{\prime}(s) \\
d(s)=b^{\prime}(s)=\frac{1}{2} a^{\prime \prime}(s)
\end{gathered}
$$

将式(16) (18)代入式(13) (15)可得

$$
\dot{\boldsymbol{q}}(s)=\boldsymbol{q}^{\prime}(s) \sqrt{a(s)}
$$

$\dddot{\boldsymbol{q}}(s)=\sqrt{a(s)}\left(\boldsymbol{q}^{\prime}(s) d(s)+3 \boldsymbol{q}^{\prime \prime}(s) b(s)+\boldsymbol{q}^{\prime \prime \prime}(s) a(s)\right)$

为得到平滑的运动轨迹, 以使动作平稳、无冲 击, 需要对速度与加速度施加约束。根据式(21)和 (22), 速度与加速度约束可表示为

$$
\begin{gathered}
\dot{\boldsymbol{q}}_{\text {min }} \leqslant \boldsymbol{q}^{\prime}(s) \sqrt{a(s)} \leqslant \dot{\boldsymbol{q}}_{\text {max }} \\
\ddot{\boldsymbol{q}}_{\text {min }} \leqslant \boldsymbol{q}^{\prime}(s) b(s)+\boldsymbol{q}^{\prime \prime}(s) a(s) \leqslant \ddot{\boldsymbol{q}}_{\max }
\end{gathered}
$$

为避免动作起始与终止时速度与加速度引起的 振动与冲击, 施加边界约束

$$
\begin{array}{ll}
a(0)=\dot{s}_{0}^{2} & a(1)=\dot{s}_{T}^{2} \\
b(0)=\ddot{s}_{0} & b(1)=\ddot{s}_{T}
\end{array}
$$

建立非线性优化模型

$$
\begin{array}{ll}
\min _{a(s), b(s)} & \int_{0_{+}}^{1} \frac{1}{\sqrt{a(s)}} \mathrm{d} s \\
\text { s.t } \quad & \dot{\boldsymbol{q}}_{\min } \leqslant \boldsymbol{q}^{\prime}(s) \sqrt{a(s)} \leqslant \dot{\boldsymbol{q}}_{\max } \\
& \ddot{\boldsymbol{q}}_{\min } \leqslant \boldsymbol{q}^{\prime}(s) b(s)+\boldsymbol{q}^{\prime \prime}(s) a(s) \leqslant \ddot{\boldsymbol{q}}_{\max } \\
a(0) & =\dot{s}_{0}^{2}, a(1)=\dot{s}_{T}^{2} \\
& b(0)=\ddot{s}_{0}, b(1)=\ddot{s}_{T} \\
\forall s \in[0,1] \\
\\
b(s)=\frac{1}{2} a^{\prime}(s) \\
a(s)>0 \\
\forall s \in\left[0_{+},{ }_{-}\right]
\end{array}
$$

由于式(28)中目标函数为凸函数 ${ }^{[15]}$, 并且所有 约束都是线性的，因此非线性优化问题为凸优化问 题, 所求得的局部最优解为全局最优解。也即, 通 过将时间最优轨迹规划问题转化为凸优化问题, 可 求得满足约束条件的全局时间最优解。

\section{3 凸优化模型求解}

对于时间最优轨迹规划的凸优化模型，需要在 一个无穷维的函数空间中搜索得到最优解, 显然这 是不可行的, 需要对函数空间进行降维 ${ }^{[16]}$ 。假定采 用一个有限维的矢量 $\boldsymbol{x}$ 表示轨迹，并以此作为规划 矢量进行规划求解得到最优解 $\boldsymbol{x}^{*}$, 则与式(10)对应 的规划模型为

$$
\begin{array}{ll}
\boldsymbol{x}^{*}=\min f(\boldsymbol{x}) \\
\text { s.t } \quad C(\boldsymbol{x}) \leqslant 0 \\
& C e q(\boldsymbol{x})=0
\end{array}
$$

由于 $\mathrm{B}$ 样条曲线具有凸包性、 $C^{k-1}$ 连续、局部 支承等优良特性，以其描述轨迹可使轨迹平滑且易 于修改与控制。轨迹可由关系式 $a(s)$ 表示, $b(s)$ 也 
可由 $a(s)$ 依式(19)间接得到, 因此选择 $\mathrm{B}$ 样条曲线 对 $a(s)$ 进行参数表示, 以有限维矢量 $\boldsymbol{X}$ 表示 $a(s)$, 进而对轨迹进行描述。

对应于离散点序列 $P$, 构造 $k$ 阶 $\mathrm{B}$ 样条曲线, 则控制顶点个数为 $n+k+1$, 节点个数为 $n+2 k+2$ 。 节点矢量 $\boldsymbol{u}$ 参照式(1)。

进行规划求解时, 需要提供 $a(s)$ 控制顶点的初 始估计值。为方便初始估计值的获取, 修改节点矢 量 $\boldsymbol{u}$ 为

$$
\begin{aligned}
\boldsymbol{u}_{x}= & {[\underbrace{0, \ldots, 0}_{k+1}, \frac{0+s_{k+1}}{2}, \ldots, \frac{s_{p}+s_{p+1}}{2}, \ldots, \frac{s_{n+k}+1}{2}, \underbrace{1, \ldots, 1}_{k+1}]=} \\
& {[\underbrace{0, \ldots, 0}_{k+1}, \bar{s}_{k+1}, \ldots, \bar{s}_{p+1}, \ldots, \bar{s}_{n+k+1}, \underbrace{1, \ldots, 1}_{k+1}] }
\end{aligned}
$$

控制顶点个数与节点个数分别加 1 , 各为 $n+k+2$ 与 $n+2 k+3$ 。

$a(s)$ 的控制顶点矢量设为

$$
\boldsymbol{c}=\left(\tilde{a}_{0}, \tilde{a}_{1}, \ldots, \tilde{a}_{n+k+1}, \tilde{a}_{n+k+2}\right)
$$

$a(s) 、 b(s)$ 与 $d(s)$ 可分别表示为

$$
\begin{gathered}
a(s)=\sum_{j=i-k}^{i} \tilde{a}_{j} N_{j}^{k}(s) \\
b(s)=\frac{1}{2} a^{\prime}(s)=\frac{1}{2} \sum_{j=i-k+1}^{i} \tilde{a}_{j}^{1} N_{j}^{k-1}(s) \\
d(s)=\frac{1}{2} a^{\prime \prime}(s)=\frac{1}{2} \sum_{j=i-k+2}^{i} \tilde{a}_{j}^{2} N_{j}^{k-2}(s)
\end{gathered}
$$

式中, $i=k, \cdots, n+k+1$; $\mathrm{B}$ 样条基函数 $N_{j}^{k}(s)$ 、 $N_{j}^{k-1}(s)$ 与控制顶点 $\tilde{a}_{j}^{1}$ 的求解参考式(3)、(4)、(6)。

$\dot{\boldsymbol{q}}(s) 、 \ddot{\boldsymbol{q}}(s) 、 \dddot{\boldsymbol{q}}(s)$ 可结合式(22) (24)与式(33) (35) 求得。

由式(26)可知

$$
\tilde{a}_{0}=a(0)=\dot{s}_{0}^{2}, \tilde{a}_{n+k+2}=a(1)=\dot{s}_{T}^{2}
$$

即, $\tilde{a}_{0}$ 与 $\tilde{a}_{n+k+2}$ 已为定值。

凸优化模型规划矢量取为

$$
\boldsymbol{x}=\left(\tilde{a}_{1}, \cdots, \tilde{a}_{n+k+1}\right)
$$

以 $\boldsymbol{x}_{p}$ 表示 $\boldsymbol{x}$ 的初始估计值, 令

$$
\boldsymbol{x}_{p}(j)=\dot{s}_{j}^{2} \approx\left[\frac{\Delta s_{j}}{\Delta t_{j}}\right]^{2} j=0,1, \cdots, n+k
$$

式中

$$
\begin{gathered}
\Delta s_{j}=s_{k+j+1}-s_{k+j} \\
\Delta t_{j}=\max \left|\frac{\boldsymbol{P}_{j+1}-\boldsymbol{P}_{j}}{\dot{\boldsymbol{q}}_{\max }}\right|
\end{gathered}
$$

得到凸优化模型的规划矢量 $\boldsymbol{x}$ 与规划矢量初始

值 $\boldsymbol{x}_{p}$ 。

原凸优化模型转化为

$$
\begin{array}{ll}
\boldsymbol{x}^{*}= & \min _{x} \int_{0_{+}}^{1_{-}} \frac{1}{\sqrt{a(s)}} \mathrm{d} s \\
\text { s.t } \quad & \dot{\boldsymbol{q}}_{\min } \leqslant \boldsymbol{q}^{\prime}(s) \sqrt{a(s)} \leqslant \dot{\boldsymbol{q}}_{\max } \\
& \ddot{\boldsymbol{q}}_{\min } \leqslant \boldsymbol{q}^{\prime}(s) b(s)+\boldsymbol{q}^{\prime \prime}(s) a(s) \leqslant \ddot{\boldsymbol{q}}_{\max } \\
& a(0)=\dot{s}_{0}^{2}, a(1)=\dot{s}_{T}^{2} \\
& b(0)=\ddot{s}_{0}, b(1)=\ddot{s}_{T} \\
& \forall s \in[0,1] \\
& b(s)=\frac{1}{2} a^{\prime}(s) \\
& a(s)>0 \\
& \forall s \in\left[0,0_{+}\right]
\end{array}
$$

此模型可采用一些成熟的优化解法进行求解, 如 SQP 法, 内点法等。

\section{4 算例仿真}

为验证所提方法的有效性, 对举高消防车进行 时间最优轨迹规划算例仿真。举高消防车各个关节 由液压缸驱动，根据液压缸的强度、受力及实际应 用需求进行计算与试验分析, 最终确定各关节的速 度及加速度约束如表 1 所示。

表 1 运动学约束

\begin{tabular}{ccccc}
\hline & \multicolumn{4}{c}{ 各个关节约束值 } \\
\cline { 2 - 5 } 号臂伸缩 & 二号臂变幅 & 二号臂伸缩 & 飞臂变幅 \\
\hline $\begin{array}{c}300 \\
\mathrm{~mm} \cdot \mathrm{s}^{-1}\end{array}$ & $\begin{array}{c}0.05 \\
\mathrm{rad} \cdot \mathrm{s}^{-1}\end{array}$ & $\begin{array}{c}240 \\
\mathrm{~mm} \cdot \mathrm{s}^{-1}\end{array}$ & $\begin{array}{c}0.12 \\
\mathrm{rad} \cdot \mathrm{s}^{-1}\end{array}$ \\
\hline 加速度 & $\begin{array}{c}250 \\
\mathrm{~mm} \cdot \mathrm{s}^{-2}\end{array}$ & $\begin{array}{c}0.08 \\
\mathrm{rad} \cdot \mathrm{s}^{-2}\end{array}$ & $\begin{array}{c}250 \\
\mathrm{~mm} \cdot \mathrm{s}^{-2}\end{array}$ & $\begin{array}{c}0.15 \\
\mathrm{rad} \cdot \mathrm{s}^{-2}\end{array}$ \\
\hline
\end{tabular}

首先，根据避障算法 ${ }^{[14]}$ 得到 4 个关节运动的离 散点序列 $P$, 并采用 $\mathrm{B}$ 样条曲线构造连续运动几何 路径。然后，以连续路径为基础，建立时间最短的 目标函数，根据表 1 施加各个关节速度与加速度约 束，并将各个关节的启动与停止的速度与加速度均 取为 0 , 进而得到时间最优轨迹规划的凸优化模型。 最后, 利用 $\mathrm{B}$ 样条曲线参数表示运动轨迹, 代入凸 优化模型, 并对模型进行离散化, 选择内点法对凸 优化模型进行求解。构造连续路径及参数表示运动 轨迹的 $\mathrm{B}$ 样条曲线阶次 $k$ 统一定为 4 , 以保证各个 关节速度、加速度及加加速度的连续性。

将伪位移 $S$ 离散为 1200 个点, 经求解得到伪速 度 $\dot{s}$ 、伪加速度 $\dddot{s}$ 及伪加加速度 $\dddot{s}$ 随 $s$ 的变化曲线如 图 2 所示。可知， $\dot{s} 、 \ddot{s}$ 及 $\dddot{s}$ 在 $s=0$ 与 $s=1$ 时均为 0 , 且当 $s \in(0,1)$ 时, 总有 $\dot{s}>0$, 与所做约定相符。 


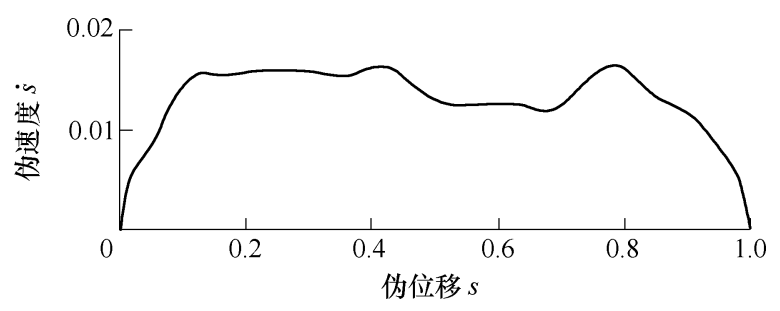

(a) $\dot{s}-s$

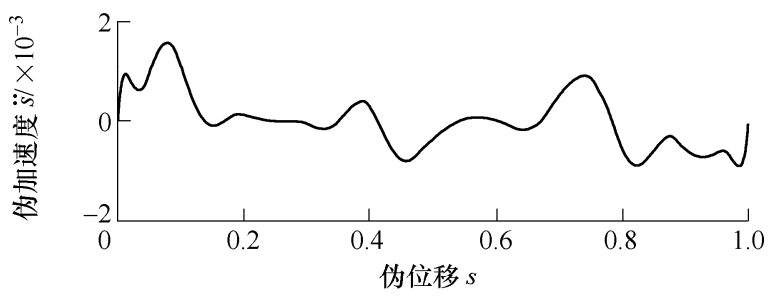

(b) $\ddot{s}-s$

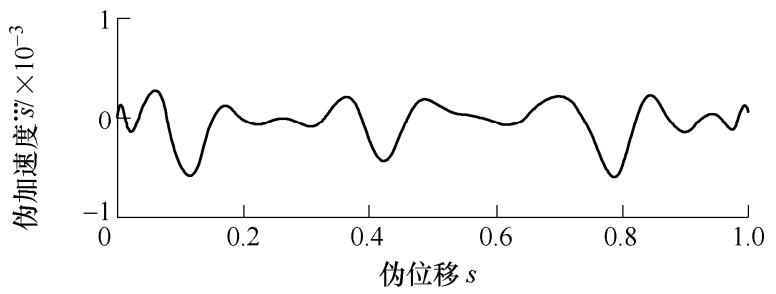

(c) $\dddot{s}-s$

图 $2 \dot{s} 、 \ddot{s}$ 与 $\dddot{s}$ 曲线图

根据式

$$
t_{f}=\int_{0}^{s_{f}} \frac{1}{\dot{s}} \mathrm{~d} s
$$

可计算任意伪位移 $s_{f}$ 对应的动作时刻 $t_{f}$ 。当 $s_{f}=1$ 时, 得到最优的总动作时间 $T=100.32 \mathrm{~s}$ 。

各个关节的位移与角位移随时间变化曲线如图 3 所示, 速度、加速度及加加速度随时间变化曲线 如图 4 6 所示。根据图中所示, 速度、加速度、加 加速度均保持变化的连续性, 并且速度、加速度始 终对时间可导, 曲线无尖峰与突变。这保证了各个 关节运动的可实现性与无冲击性。同时, 由于速度、 加速度、加加速度的初始值与终止值均为 0 , 各个

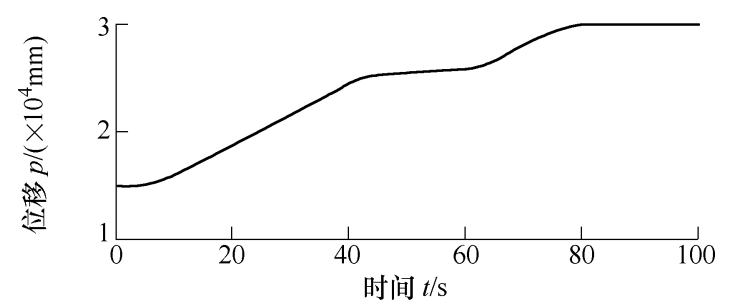

(a) 一号臂伸缩位移

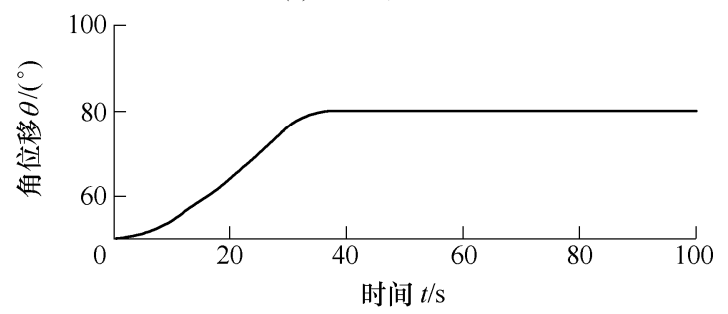

(b) 二号臂变幅角位移

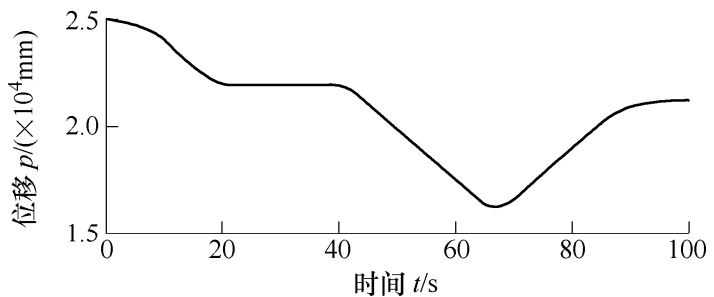

(c) 二号臂伸缩位移

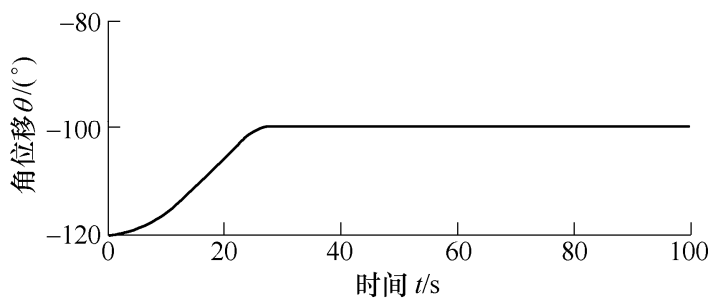

(c) 飞臂变幅角位移

图 3 各个关节位移与角位移

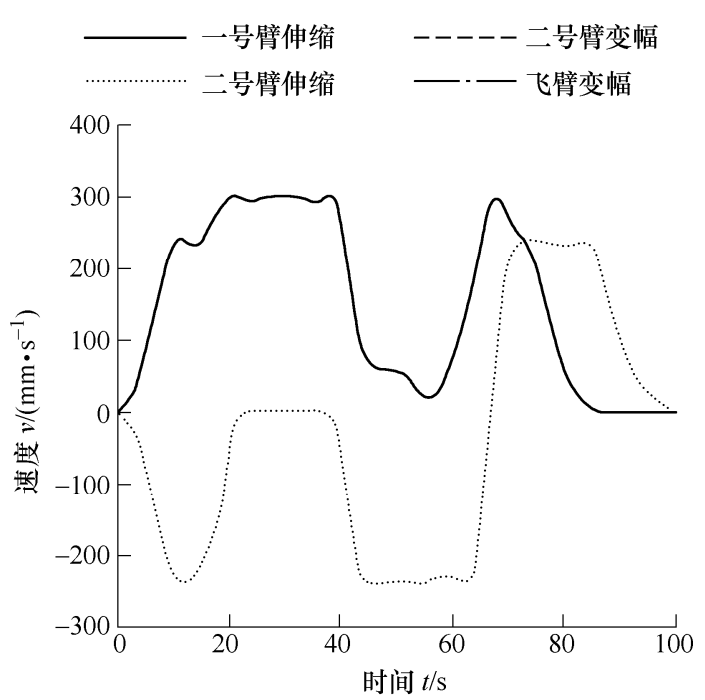

(a)一号臂 \&二号臂伸缩速度

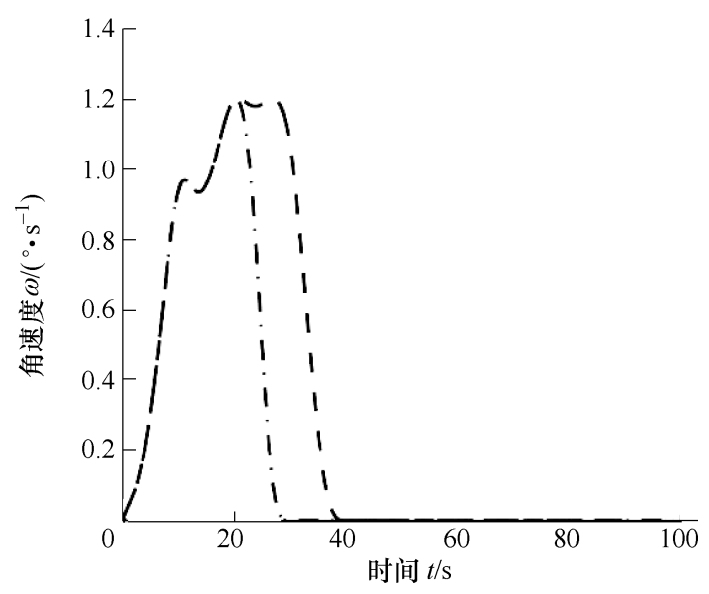

(b) 二号臂 \& 飞臂变幅角速度

图 4 各个关节速度与角速度

关节在运动起始与运动终止时可获得较好的运动与 动力性能, 速度与驱动力均可较为平缓地完成从无 至有或从有至无的切换。尤其当运动终止时，关节 无残余动能, 可有效避免臂架振动。关节的速度极 
限也得到充分利用, 在启动与停止的中间阶段至少 有一个关节保持接近约束值的速度进行动作。

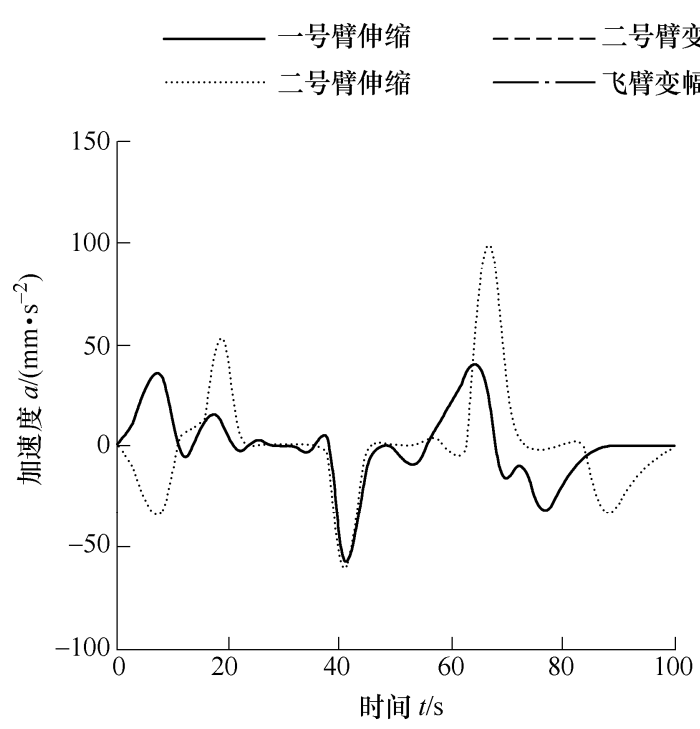

(a)一号臂 \& 二号臂伸缩加速度

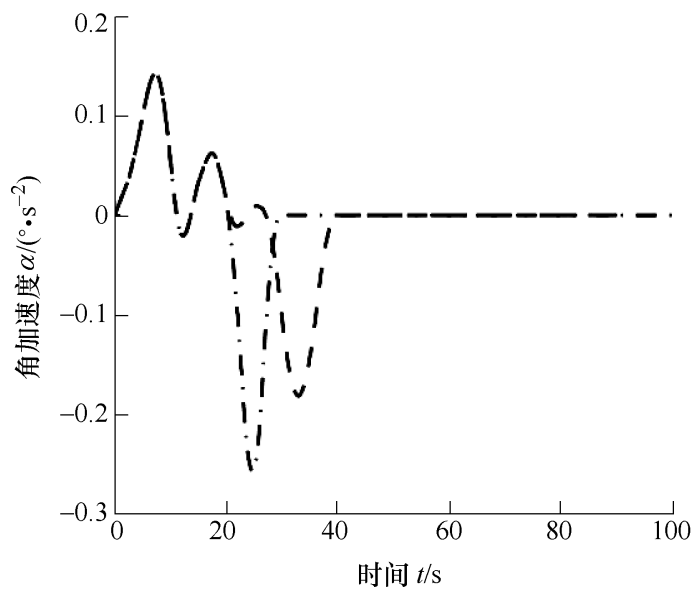

(b) 二号臂 \& 飞臂变幅角加速度

图 5 各个关节加速度与角加速度
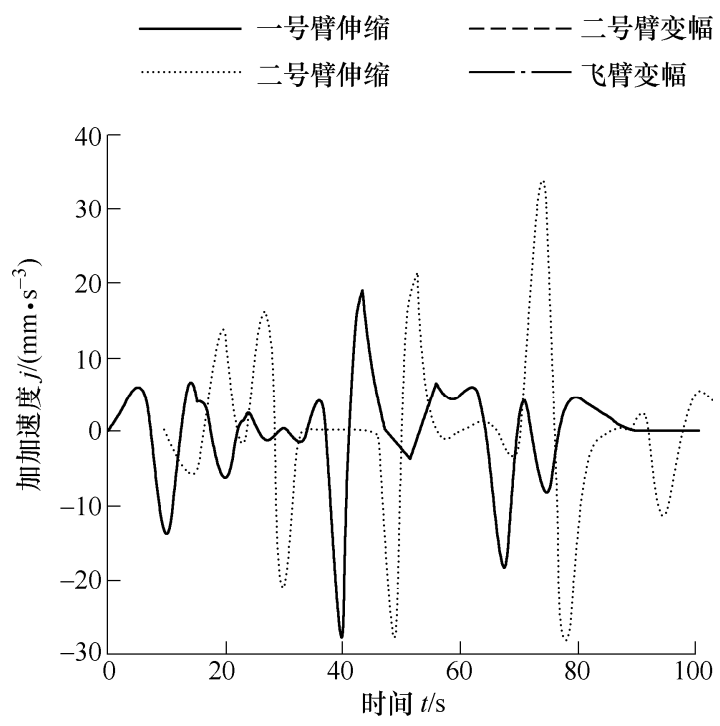

(a) 一号臂 \&二号臂伸缩加加速度

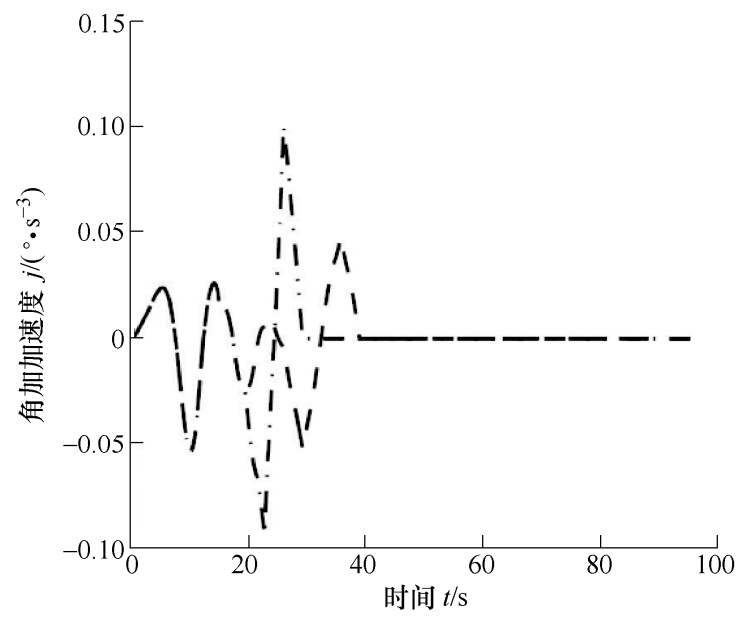

(b) 二号臂 \& 飞臂变幅角加加速度

图 6 各个关节加加速度与角加加速度

各个关节对应的运动极限值如表 2 所示, 经过 与表 1 数据对比可得各个关节速度、加速度约束均 得到满足。加速度、加加速度保持在一个约束范围 内, 表示速度、加速度的变化率小, 动作平稳性便 得到保证。

表 2 运动极限值

\begin{tabular}{ccccc}
\hline & \multicolumn{4}{c}{ 各个关节约束值 } \\
\cline { 2 - 5 } & 一号臂伸缩 & 二号臂变幅 & 二号臂伸缩 & 飞臂变幅 \\
\hline \multirow{2}{*}{ 速度 } & $\begin{array}{c}300 \\
\mathrm{~mm} \cdot \mathrm{s}^{-1}\end{array}$ & $\begin{array}{c}0.0209 \\
\mathrm{rad} \cdot \mathrm{s}^{-1}\end{array}$ & $\begin{array}{c}240 \\
\mathrm{~mm} \cdot \mathrm{s}^{-1}\end{array}$ & $\begin{array}{c}0.0209 \\
\mathrm{rad} \cdot \mathrm{s}^{-1}\end{array}$ \\
\hline \multirow{2}{*}{ 加速度 } & 57.77 & 0.0032 & 101.91 & 0.0045 \\
& $\mathrm{~mm} \cdot \mathrm{s}^{-2}$ & $\mathrm{rad} \cdot \mathrm{s}^{-2}$ & $\mathrm{~mm} \cdot \mathrm{s}^{-2}$ & $\mathrm{rad} \cdot \mathrm{s}^{-2}$ \\
\hline
\end{tabular}

另外, 计算得知, 当将伪位移 $S$ 分别离散为 100 、 500 及 1200 个点时, 所得最优时间依次递减, 分别 为 $108.59 \mathrm{~s} 、 104.95 \mathrm{~s}$ 及 $100.32 \mathrm{~s}$ 。可知, 伪位移离 散点越密集, 则与原始凸优化模型越接近, 所得最 优时间越短, 规划结果也越理想。但是, 密集程度 越高, 相应的计算耗时也越高。应用情况不同, 对 运动位置误差与响应时间的要求不同。因此, 应根 据应用情况的运动误差与响应时间设定目标, 设定 伪位移离散点个数的变化区间, 通过算法测试确定 合适的伪位移离散点个数。

\section{5 结论}

（1）针对具有多种关节形式的举高消防车，提 出一种时间最优轨迹规划方法，综合考虑动作时间 与动作平稳性, 以各个关节运动离散点为基础, 利 用凸优化理论建立数学模型。此类模型形式统一, 结构简单, 可方便设定目标函数并施加约束, 适应 性与可行性强。

(2) 利用 $B$ 样条曲线得出运动轨迹的参数表 
示, 以内点法对凸优化模型进行求解, 得到平滑的 时间最优运动轨迹。对于凸优化模型, 局部最优解 为全局最优解, 因此所求得的运动轨迹为全局性的 时间最优运动轨迹。

(3) 应用所得运动轨迹于举高消防车, 可缩短 举高消防车的作业时间, 提高作业效率, 同时由于 臂架动作平稳、无冲击, 消防与救援作业更加可靠, 末端平台上人员的安全也得到保障。

\section{参 考 文 献}

[1] RAJAN V. Minimum time trajectory planning[C]// IEEE International Conference on Robotics and Automation. Proceedings. Piscataway: IEEE, 1985: 759-764.

[2] 钱东海, 谭伟, 赵锡芳. 基于 B 样条路径的机器人时间 最优轨迹规划 $[\mathrm{J}]$. 上海交通大学学报, 1998, 32(12): 31-35.

QIAN Donghai, TAN Wei, ZHAO Xifang. Time optimum trajectory planning for robots based on B-spline path[J]. Journal of Shanghai Jiao Tong University, 1998, 32(12): 31-35.

[3] CHO B H, CHOI B S, LEE J M. Time-optimal trajectory planning for a robot system under torque and impulse constraints[J]. International Journal of Control Automation \& Systems, 2006, 4(1): 10-16.

[4] BIANCO C, PIAZZI A. Minimum-time trajectory planning of mechanical manipulators under dynamic constraints[J]. International Journal of Control, 2002, 75(13): 967-980.

[5] KHOUKHI A, BARON L, BALAZINSKI M, et al. A hierarchical neuro-fuzzy system to near optimal-time trajectory planning of redundant manipulators[J]. Engineering Applications of Artificial Intelligence, 2008, 21(7): 974-984.

[6] GAO M, DING P, YANG Y. Time-optimal trajectory planning of industrial robots based on particle swarm optimization[C]// International Conference on Instrumentation \& Measurement. Piscataway: IEEE, 2015: 1934-1939.

[7] SURYAWAN F, DONÁ J, SERON M. Minimum-time trajectory generation for constrained linear systems using flatness and B-splines[J]. International Journal of Control, 2011: 1565-1585.

[8] KAMALAPURKAR R, DINH H, BHASIN S, et al. Approximate optimal trajectory tracking for continuous-time nonlinear systems[J]. Automatica, 2015,
51: $40-48$.

[9] LIU H, LAI X, WU W. Time-optimal and jerk-continuous trajectory planning for robot manipulators with kinematic constraints[J]. Robotics and Computer-integrated Manufacturing, 2013, 29(2): 309-317.

[10] REYNOSO-MORA P, CHEN W, TOMIZUKA M. On the time-optimal trajectory planning and control of robotic manipulators along predefined paths $[\mathrm{C}] / /$ American Control Conference. Piscataway: IEEE, 2013: 371-377.

[11] 滕儒民. 高空作业车臂架系统快速设计及其运动规划 研究[D]. 大连: 大连理工大学, 2012.

TENG Rumin. Rapid design and motion planning of the boom system for the aerial work platform[D]. Dalian: Dalian University of Technology, 2012.

[12］袁合. 折臂式高空作业车轨迹规划与控制研究[D]. 大 连: 大连理工大学, 2013.

YUAN He. Track programming and controlling of folding boom aerial working platform[D]. Dalian: Dalian University of Technology, 2013.

[13] 王欣, 高凌羽, 黄兆秋, 等. 直臂高空作业车船体喷涂 轨迹控制研究与仿真 $[\mathrm{J}]$. 系统仿真学报, 2016, 28(2): 404-409.

WANG Xin, GAO Lingchong, HUANG Zhaoqiu, et al. Research and simulation on trajectory control of telescopic boom aerial work platform for hull spray painting[J]. Journal of System Simulation，2016，28(2): 404-409.

[14] 滕儒民，贺浩，项慧，等. 基于启发式路径搜索的高空 作业车避障轨迹规划[J]. 机械工程学报, 2013, 49(10): 194-198.

TENG Rumin, HE Hao, XIANG Hui, et al. Trajectory planning with obstacles considered for aerial work platform based on heuristic path-searching[J]. Journal of Mechanical Engineering, 2013， 49(10): 194-198.

[15] VERSCHEURE D, DEMEULENAERE B, SWEVERS J, et al. Time-optimal path tracking for robots: A convex optimization approach[J]. IEEE Transactions on Automatic Control, 2009, 54(10): 2318-2327.

[16] REITER A. Time-optimal trajectory planning for redundant robots[M]. Wiesbaden: Springer Verlag, 2016.

作者简介: 滕儒民, 男, 1973 年出生, 博士, 副教授。主要研究方向为 工程机械及特种车辆新产品开发及设计方法。

E-mail: tengrumin@163.com

李玉金金(通信作者), 男, 1990 年出生。主要研究方向为工程机械及特种 车辆新产品开发及设计方法。

E-mail: liyuxin_2016@163.com 